\title{
Quantifying seasonal phytoplankton oscillations in the global offshore ocean
}

\author{
J. A. Rudjakov* \\ Laboratory of Plankton Ecology and Distribution, P. P. Shirshov Institute of Oceanology, Russian Academy of Sciences, \\ 23 Krasikov St., Moscow 117218, Russia
}

\begin{abstract}
Monthly medians of phytoplankton pigment concentration in the Global Data Set of the Coastal Zone Color Scanner and direct chlorophyll measurements at the Japanese Antarctic Station SYOWA were used to study the global scale pattern of seasonal phytoplankton oscillations between $55^{\circ} \mathrm{N}$ and $69^{\circ} \mathrm{S}$. Using periodic regression, sine and cosine amplitudes for the annual and the semiannual harmonic components were estimated and the amplitudes expressed as polynomial functions of geographical latitude. At latitudes $>50^{\circ}$, seasonal oscillations run in approximately opposite phases in the Northern and Southern Hemispheres, with hemispheric summer maxima and winter minima. The same phase reversal is clearly seen between $10^{\circ}-40^{\circ} \mathrm{N}$ and $10^{\circ}-40^{\circ} \mathrm{S}$, but the maxima are observed in hemispheric winter, and the minima in summer. Between $10^{\circ} \mathrm{N}$ and $10^{\circ} \mathrm{S}$, with 2 maxima of solar radiation, 2 pigment maxima per year are observed, and the same 2 maxima presumably occur near $50^{\circ} \mathrm{N}$ and $50^{\circ} \mathrm{S}$, where the phase of the oscillation abruptly changes. In spite of the intentionally simplistic character of the approach, the derived pattern retains and quantifies many features of seasonal phytoplankton changes at different latitudes known from the literature
\end{abstract}

KEY WORDS: Phytoplankton · Pigment concentration - Seasonalıty - Global scale $\cdot$ Coastal Zone Color Scanner

\section{INTRODUCTION}

The oceanic phytoplankton abundance changes greatly not only in space but also in time. The general pattern of phytoplankton distribution on a global scale was assessed using both biomass evaluation based on cell counts (Semina 1977) and pigment concentrations estimated by remote sensing for numerous areas in the offshore ocean (Banse \& English 1994). The patterns of ocean-wide seasonality of phytoplankton were also described by remote sensing for latitudinal bands, including the shelves (Yoder et al. 1993). Nevertheless, in modelling oceanic productivity and in making rough estimates of global ecological relations, these rules need to be expressed mathematically. Recently, an expression was constructed for latitude-dependent seasonal oscillations of mesozooplankton (Rudjakov et al. 1995). To extract seasonal variability from zoo-

\footnotetext{
-Present address: 151 Glen St., Somerville, Massachusetts 02145, USA
}

plankton time series, the harmonic least squares fit was used. The derived estimates of the sine and cosine amplitudes of annual and semiannual variability were expressed as polynomial functions of latitude. This article applies the same statistics to annual cycles of phytoplankton.

\section{MATERIALS AND METHODS}

The main bulk of the data was obtained from panels illustrating seasonal changes of median pigment concentration at 48 offshore sites derived by Banse \& English (1994, Figs. 3 to 7) from the monthly mean pigment arrays in the Global Data Set of the Coastal Zone Color Scanner between $55^{\circ} \mathrm{N}$ and $53^{\circ} \mathrm{S}$. The sites were chosen to be '... seemingly typical as well as singular ... in a framework formed by the major oceanic convergences' (Banse \& English 1994, p. 7330). This data set of estimates of near-surface pigment was supplemented by in situ chlorophyll measurements at the Japanese Antarctic near-shore Station SYOWA, at 


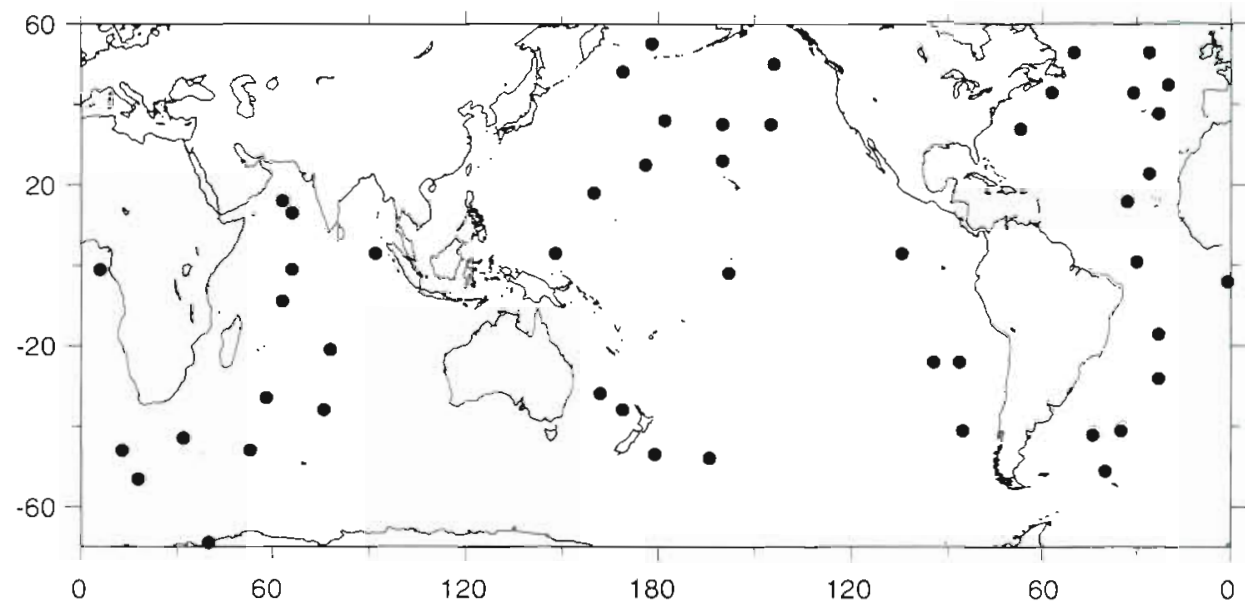

Fig. 1. Observation sites

$69^{\circ} \mathrm{S}$ (Fukuchi et al. 1985). From the latter, only 322 measurements between 3 and $26 \mathrm{~m}$ depth were used, because, judging from abrupt temperature changes, the upper layer was subject to quite irregular dilution by melt water during summer, and the deeper layers were not sampled regularly. The observation sites are shown in Fig. 1.

The log-transformed (to base 10) monthly medians in each region were fitted to a periodic regression (Bliss 1958) with 2 harmonic terms, which have annual and semiannual periods respectively $(2$ higher harmonics were also tested, but their influence on the residual mean squares proved to be insignificant):

$$
\begin{aligned}
\log (B)= & a+b \cos (\pi t / 6)+c \sin (\pi t / 6) \\
& +d \cos (\pi t / 3)+e \sin (\pi t / 3)
\end{aligned}
$$

where $B$ is the pigment concentration; $a$ is the mean $\log$ of $B$ (antilog of $a$ is the annual geometric mean pigment concentration $B^{\prime}$ ); and $b, c, d$ and $e$ are the cosine and sine amplitudes. The coefficients $b, c, d$ and $e$ are invariable with the concentration unit, as $\log (B)-a=$ $\log \left(B / B^{\prime}\right)$, whose antilog is the factor by which the biomass value at time $t$ exceeds the annual mean biomass level. Time $t$ was equal to month minus 1 plus (day divided by 32 ). For example, for 31 December, $t=$ $12-1+31 / 32=11.97$ and for 1 January, $t=1-1+1 / 32$ $=0.03$. For a monthly median, $t$ is equal to month minus 1 plus 15/32. The multiple regression was fitted such that $\log (B)$ was the dependent variable. and $\cos (\pi t / 6), \sin (\pi t / 6), \cos (\pi t / 3)$, and $\sin (\pi t / 3)$ were independent variables.

To construct a global scale pattern of seasonal oscillations, sine and cosine amplitudes $b, c, d$, and $e$ of Eq. (1) were considered as functions of latitude using a polynomial fitting:

$$
P=k_{0} L^{0}+k_{1} L^{1}+k_{2} L^{2}+k_{3} L^{3}+k_{4} L^{4}
$$

where $L$ is latitude (degrees); and $P$ denotes $b, c, d$, or e from Eq. (1)

\section{RESULTS AND DISCUSSION}

The quality of fit of seasonal cycle regression is measured by the statistic $100 \mathrm{r}^{2}$ which, in this context, represents the percentage of the variance of pigment concentration that is explained by the regression model described by Eq. (1). The periodic regression with 2 harmonic terms accounts for 21 to $98 \%$ of the total variance of pigment values (Table 1 ). The $100 \mathrm{r}^{2}$ does not show an apparent relation to the latitude and on average equals $71 \%$.

The amplitude calculations (Table 1) show that the oscillation amplitudes for the first harmonic component $A l=\sqrt{ }\left(b^{2}+c^{2}\right)$ and for the second harmonic component $A 2=\sqrt{ }\left(d^{2}+e^{2}\right)$ are not clearly related to the latitude, contrary to the first harmonic component of mesozooplankton biomass oscillations (Rudjakov et al. 1995). Nevertheless, some of the terms of polynomial fitting of the latitudinal changes in the $b, c, d$, and e coefficients proved to be significant at $p<0.05$ or even at $p<0.001$. For a better visualization of the pattern of seasonal change of phytoplankton at different latitudes, a 2-step approach was chosen.

As the first step, all terms of Eq. (2) were held, without regard to their significance:

$$
\begin{aligned}
b= & 0.0382+0.00904 \cdot L-0.000028 \cdot L^{2} \\
& -3.906 \times 10^{-6} \cdot L^{3}+4.533 \times 10^{-11} \cdot L^{4} \\
c= & -0.0918+0.00573 \cdot L+0.000070 \cdot L^{2} \\
& -2.542 \times 10^{-6} \cdot L^{3}-1.636 \times 10^{-8} \cdot L^{4} \\
d= & 0.0533-0.00109 \cdot L-0.000076 \cdot L^{2} \\
& +3.766 \times 10^{-7} \cdot L^{3}+1.780 \times 10^{-8} \cdot L^{4} \\
e= & 0.1077-0.00042 \cdot L-0.000123 \cdot L^{2} \\
& -9.389 \times 10^{-8} \cdot L^{3}+2.961 \times 10^{-8} \cdot L^{4}
\end{aligned}
$$

The quality of the fit can be visually judged from Figs. $2 \mathrm{a}-\mathrm{d}$ where the usual data noise of a time series and the dissimilarity in the longitude depen- 
Table 1 Oscillation cosine and sine amplitudes of the first $(b, c, A 1)$ and of the second $(d, e, A 2)$ harmonics, and $100 r^{2}$ values. $L$ : latitude (south negative), $L_{0}$ : longitude (west negative)

\begin{tabular}{|c|c|c|c|c|c|c|c|c|}
\hline$L$ & $L_{0}$ & $b$ & c & Al & $d$ & e & $A 2$ & $100 r^{2}$ \\
\hline 55 & 178 & -0.1228 & 0.0128 & 0.123 & 0.1860 & 0.2201 & 0.288 & 24.23 \\
\hline 53 & -26 & -0.2647 & -0.1413 & 0.300 & -0.1721 & 0.0525 & 0.180 & 42.98 \\
\hline 53 & -50 & -0.2000 & -0.1233 & 0.235 & -0.1313 & -0.2269 & 0.262 & 73.53 \\
\hline 50 & -144 & -0.0732 & -0.1977 & 0.211 & -0.0071 & -0.0928 & 0.093 & 88.60 \\
\hline 48 & 169 & 0.0669 & -0.3083 & 0.315 & -0.1016 & -0.1806 & 0.207 & 74.09 \\
\hline 45 & -20 & -0.1498 & 0.0278 & 0.152 & 0.0201 & -0.0785 & 0.081 & 52.87 \\
\hline 43 & -31 & 0.0565 & 0.0482 & 0.074 & -0.0139 & -0.1292 & 0.130 & 26.59 \\
\hline 43 & -57 & 0.0898 & 0.0560 & 0.106 & -0.2132 & -0.0334 & 0.216 & 70.20 \\
\hline 38 & -23 & 0.2182 & 0.2087 & 0.302 & -0.0162 & 0.0089 & 0.018 & 98.10 \\
\hline 36 & -178 & 0.2470 & 0.2308 & 0.338 & 0.0064 & -0.0713 & 0.072 & 89.63 \\
\hline 35 & -145 & 0.2469 & 0.1717 & 0.301 & 0.0894 & 0.1017 & 0.135 & 93.39 \\
\hline 35 & -160 & 0.3034 & 0.2445 & 0.390 & 0.0924 & -0.0401 & 0.101 & 97.60 \\
\hline 34 & -67 & 0.2411 & 0.1294 & 0.274 & -0.0246 & -0.0726 & 0.077 & 97.56 \\
\hline 26 & -160 & 0.1317 & 0.0313 & 0.135 & 0.0022 & 0.0367 & 0.037 & 96.07 \\
\hline 25 & 176 & 0.0550 & 0.0358 & 0.066 & 0.0325 & 0.0644 & 0.072 & 93.49 \\
\hline 23 & -26 & 0.0508 & -0.0244 & 0.056 & 0.0122 & -0.0223 & 0.025 & 88.85 \\
\hline 18 & 160 & 0.0314 & 0.0056 & 0.032 & 0.0214 & 0.0023 & 0.022 & 48.18 \\
\hline 16 & -33 & 0.1484 & 0.0207 & 0.150 & 0.0318 & 0.0067 & 0.032 & 80.57 \\
\hline 16 & 63 & 0.1740 & -0.3232 & 0.367 & -0.1390 & 0.3454 & 0.372 & 79.37 \\
\hline 13 & 66 & 0.2369 & -0.3103 & 0.390 & -0.1914 & 0.2395 & 0.307 & 96.35 \\
\hline 3 & 148 & 0.0096 & -0.0269 & 0.029 & 0.0553 & 0.0497 & 0.074 & 68.71 \\
\hline 3 & -104 & 0.0354 & 0.0038 & 0.036 & 0.0343 & 0.0070 & 0.035 & 41.92 \\
\hline 3 & 92 & 0.0605 & -0.1732 & 0.183 & -0.0261 & 0.1348 & 0.137 & 97.35 \\
\hline 1 & -30 & -0.0449 & -0.0848 & 0.096 & 0.0375 & 0.0420 & 0.056 & 34.07 \\
\hline-1 & 66 & 0.0988 & 0.0085 & 0.099 & -0.0046 & 0.0418 & 0.042 & 64.62 \\
\hline-1 & 6 & 0.1982 & -0.0621 & 0.208 & 0.2936 & 0.2799 & 0.406 & 87.61 \\
\hline-2 & -158 & -0.0574 & 0.0245 & 0.062 & 0.0806 & 0.0465 & 0.093 & 55.02 \\
\hline-4 & -1 & -0.1594 & -0.2583 & 0.304 & 0.0633 & 0.2217 & 0.231 & 93.50 \\
\hline-9 & 63 & -0.0798 & -0.1168 & 0.141 & -0.0047 & 0.0984 & 0.099 & 74.01 \\
\hline-17 & -23 & -0.0265 & -0.0060 & 0.027 & -0.0016 & -0.0110 & 0.011 & 30.87 \\
\hline-21 & 78 & 0.2321 & -0.1119 & 0.258 & 0.2791 & -0.0491 & 0.283 & 52.75 \\
\hline-24 & -86 & -0.1527 & -0.0837 & 0.174 & 0.0021 & 0.0572 & 0.057 & 88.73 \\
\hline-24 & -94 & -0.0981 & -0.0416 & 0.107 & 0.0167 & 0.0377 & 0.041 & 86.46 \\
\hline-28 & -23 & -0.1957 & -0.1249 & 0.232 & 0.0850 & -0.0097 & 0.086 & 75.42 \\
\hline-32 & 162 & -0.2439 & -0.2024 & 0.317 & 0.0061 & -0.0373 & 0.038 & 96.72 \\
\hline-33 & 58 & -0.2083 & -0.2331 & 0.313 & 0.0227 & 0.0433 & 0.049 & 84.06 \\
\hline-36 & 169 & -0.2029 & -0.2561 & 0.327 & -0.0737 & -0.0318 & 0.080 & 86.61 \\
\hline-36 & 76 & -0.1848 & -0.1798 & 0.258 & 0.0825 & -0.0102 & 0.083 & 90.63 \\
\hline-41 & -35 & -0.0182 & -0.0882 & 0.090 & 0.0238 & 0.0173 & 0.029 & 20.78 \\
\hline-41 & -85 & -0.2246 & -0.0595 & 0.232 & -0.0581 & 0.0222 & 0.062 & 86.46 \\
\hline-42 & -44 & -0.2792 & -0.0576 & 0.285 & 0.0110 & 0.0136 & 0.017 & 79.63 \\
\hline-43 & 32 & 0.0607 & 0.1175 & 0.132 & -0.1172 & 0.0459 & 0.126 & 79.71 \\
\hline-46 & 13 & -0.1117 & -0.1474 & 0.185 & 0.1162 & 0.1886 & 0.222 & 37.00 \\
\hline-46 & 53 & -0.1131 & -0.2669 & 0.290 & -0.1003 & 0.0924 & 0.136 & 40.61 \\
\hline-47 & 179 & 0.0794 & 0.0259 & 0.084 & -0.0386 & 0.0769 & 0.086 & 21.56 \\
\hline-48 & -164 & 0.0695 & 0.0931 & 0.116 & -0.1120 & -0.0185 & 0.114 & 68.48 \\
\hline-51 & -40 & 0.1436 & 0.0722 & 0.161 & 0.0157 & -0.0470 & 0.050 & 48.92 \\
\hline-53 & 18 & -0.2195 & -0.0242 & 0.221 & 0.0029 & -0.0286 & 0.029 & 90.22 \\
\hline-69 & 40 & 0.6160 & 0.3511 & 0.709 & 0.0658 & 0.2270 & 0.236 & 55.94 \\
\hline
\end{tabular}

dence of phytoplankton seasonality in different oceans show up. In contrast to zooplankton amplitude changes (Rudjakov et al. 1995), the latitude-dependent sine and cosine amplitude changes of phytoplankton pigment have a quite similar shape. The topography of the surface, specified by Eq. (1) with $b$, $c, d$, and e substituted by Eqs. (3), (4), (5) \& (6), is given in Fig. 3.
As the second step, only the polynomial terms significant at $\mathrm{p}<0.05$ were kept:

$$
\begin{aligned}
& b=0.0030+0.00872 \cdot L-3.710 \times 10^{-6} \cdot L^{3} \\
& c=-0.0472+0.00520 \cdot L-2.267 \times 10^{-6} \cdot L^{3} \\
& d=0 \text { (all terms are insignificant) } \\
& e=0.1077-0.000123 \cdot L^{2}+3.295 \times 10^{-8} \cdot L^{4}
\end{aligned}
$$



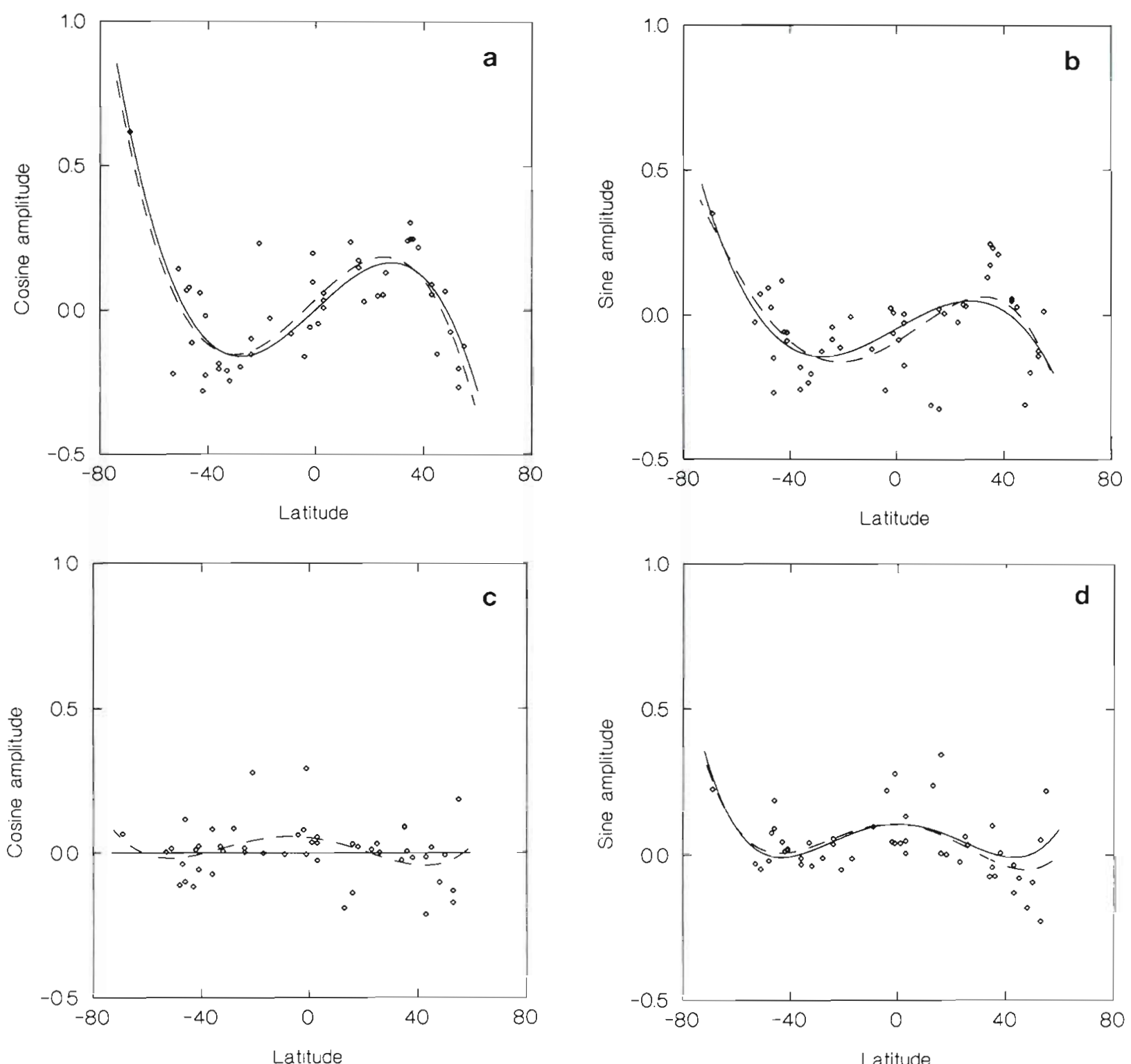

Fig. 2. Cosine and sine amplitudes versus latitude. (a) First harmonic cosine; (b) first harmonic sine; (c) second harmonic cosine; (d) second harmonic sine; (— ) approximation with Eqs. (7) to (10); ( $~-~-)$ approximation with Eqs. (3) to (6)

The goodness of fit of the polynomial Eqs. (3) to (10) can be judged from the data listed in Table 2 . With the exception of the cosine amplitude of the second harmonic term, longitude-dependent changes in amplitudes are highly significant, although the fitting of the first harmonic term is considerably better than that of the second term.

The regression lines described by Eqs. (7) to (10) are shown in Figs. 2a-d to facilitate the comparison with those described by Eqs. (3) to (6). The topography of the surface specified by Eq. (1) with $b, c, d$, and e substituted by Eqs. (7), (8), (9), and (10) is given in Fig. 4.

The comparison between the dashed and solid lines in Fig. $2 \mathrm{a}-\mathrm{d}$ and between Figs. 3 \& 4 shows that nei-
Table 2. Goodness of fit of polynomial regressions Eqs. (3) to (10) expressed as percent of the variance explained by the regression $\left(100 \mathrm{r}^{2}\right)$, the ratio of the regression and residual mean squares $(F)$, and the probability value $(p)$

\begin{tabular}{|cccc|}
\hline Eq. no & $100 \mathrm{r}^{2}$ & $F$ & $p$ \\
\hline 3 & 60.30 & 16.33 & $<0.001$ \\
7 & 57.68 & 30.66 & $<0.001$ \\
4 & 34.34 & 5.62 & 0.001 \\
8 & 30.69 & 9.96 & $<0.001$ \\
5 & 11.35 & 1.38 & $>0.05$ \\
9 & 0.0 & (not calculated) \\
6 & 29.00 & 4.39 & $<0.01$ \\
10 & 25.28 & 7.61 & 0.001 \\
\hline
\end{tabular}


ther the regression lines nor the resulting patterns differ substantially, except in small details whose significance is very problematic. The general pattern is quite stable and can be easily recognized even if only the first harmonic terms are kept. At latitudes $>50^{\circ}$, seasonal oscillations run in opposite phases in the Northern and Southern Hemispheres, with hemispheric summer maxima and winter minima. The same phase reversal is clearly seen between $10^{\circ}-40^{\circ} \mathrm{N}$ and $10^{\circ}-40^{\circ} \mathrm{S}$, but now the maxima are observed in the hemispheric winter, and the minima in summer. Between $10^{\circ} \mathrm{N}$ and $10^{\circ} \mathrm{S}, 2$ maxima per year occur, and the same can presumably be observed near latitudes $50^{\circ} \mathrm{N}$ and $50^{\circ} \mathrm{S}$, where the phase of the oscillation abruptly changes.

Judging from Fig. $2 a-d$, the sine and cosine amplitudes of the seasonal pigment oscillations at the near-shore station SYOWA (at $69^{\circ} \mathrm{S}$ ) are in line with the general trend of latitude-dependent amplitude changes in offshore oceanic regions. They show such good consistency with this trend that they change the polynomial coefficients $b, c, d$, and $e$ of Eqs. (3) to (10), derived only from the offshore data, only insignificantly. This results in practically no changes in Figs. 3 \& 4. It may simply be a coincidence, of course, and the data are certainly insufficient for more general conclusions, but this case shows that seasonal changes of phytoplankton abundance may be quite similar in some near- and offshore regions of the same latitude range.

In spite of the heuristic character of the approach, the derived pattern retains and quantifies many features of seasonal phytoplankton changes at different latitudes reviewed by Heinrich (1993) and traced and mapped anew by Banse \& English (1994). Thus, hopefully, the formulae may be useful in oceanic ecosystem modelling. In comparison with mesozooplankton biomass seasonal oscillations (Rudjakov et al. 1995), the picture developed here might seem too complicated to some. The only evidence of its reality may be seen in the general pattern of seasonal insolation changes (Holmes 1957, Fig. 2): the period of sea-

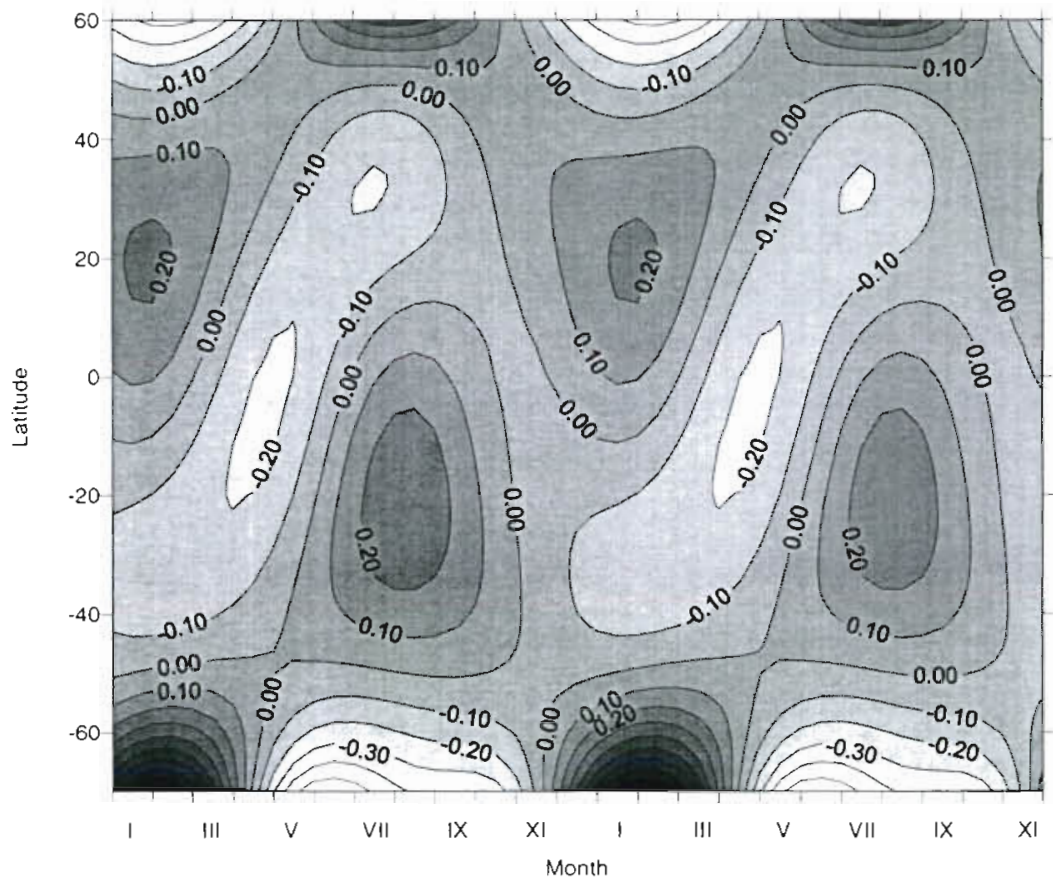

Fig. 3. Logarithmic (base 10) oscillations of phytoplankton pigment at different latitudes (south negative) relative to the mean level; approximated with Eqs. (3) to (6)

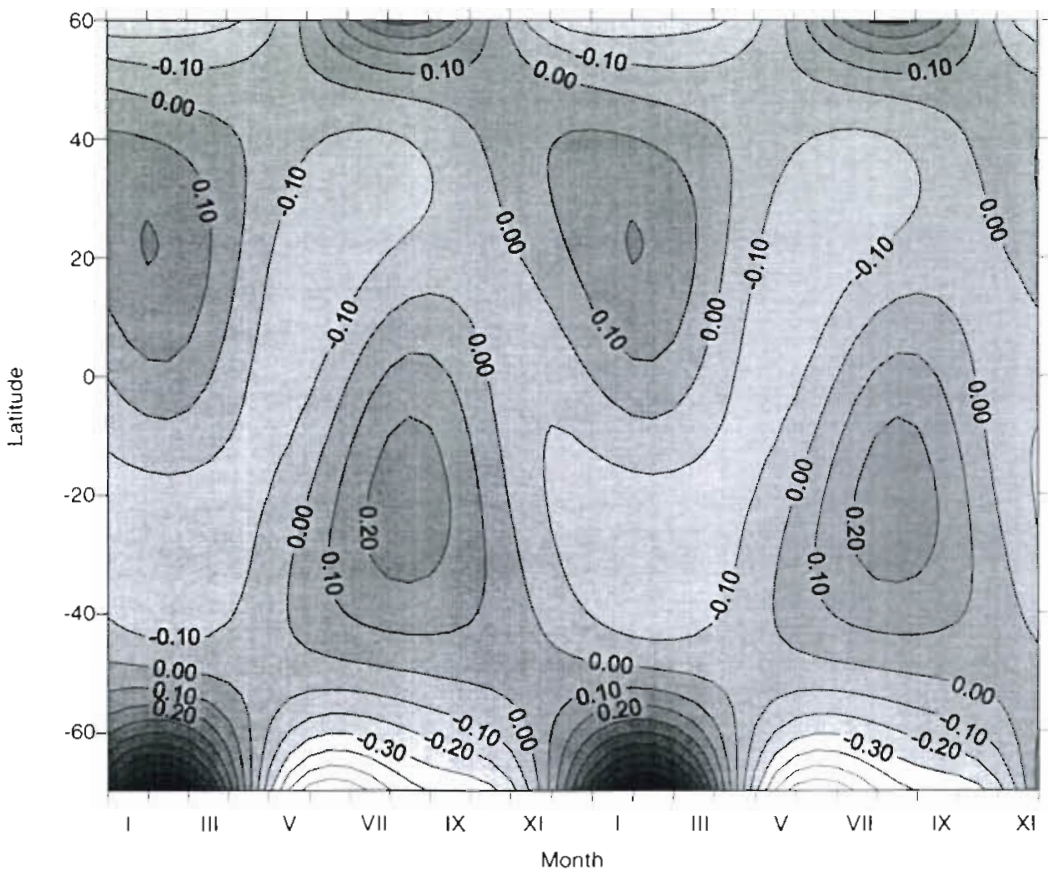

Fig. 4. Logarithmic (base 10) oscillations of phytoplankton pigment at different latitudes (south negative) relative to the mean level; approximated with Eqs. (7) to (10) 
Table 3. Months ( 1 = January, etc.) of maximal (Max) and minimal (Min) abundance values of phytoplankton pigment (Phyto) and mesozooplankton (ZOo) at different latitudes $(L)$ (south negative)

\begin{tabular}{|rcccc|}
\hline \multirow{2}{*}{$L$} & \multicolumn{2}{c}{ Max } & \multicolumn{2}{c}{ Min } \\
& Phyto & Zoo & Phyto & Zoo \\
\hline 60 & $7-8$ & $6-7$ & $1-2$ & $12-1$ \\
40 & $1-3$ & $5-6$ & 7 & 11 \\
20 & $1-2$ & 5 & $5-6$ & 11 \\
0 & $1-2,8-9$ & 11 & $4-5,11-12$ & 5 \\
-20 & $7-8$ & 12 & $3-4$ & 6 \\
-40 & $7-8$ & 1 & $1-2$ & 7 \\
-70 & $1-2$ & $2-3$ & $6-7$ & $8-9$ \\
\hline
\end{tabular}

sonal solar radiation change in the tropics is close to $6 \mathrm{mo}$, with the maxima approximately in March and September and the minima in June and December. The coincidence is very rough, and one can only say that the seasonal oscillations of insolation and of pigment concentration run approximately in the same phase. Since light does not limit the rate of primary production at low latitudes, it is unlikely itself to determine the pigment concentration in the approximately upper third of the photic zone, where the satellite data are obtained. Instead, the seasonal change of insolation must be considered as a clue for more involved processes, physical as well as biological. The correspondence between daily insolation and pigment concentration values holds also for the higher latitudes covered by this study ( 1 maximum each in the middle of summer, the pigment maxima being 1 mo later than the maxima of insolation). One could not expect a better coincidence in the case of so complicated a phenomenon as time changes of phytoplankton concentrations, which depend not only on physical and chemical processes affecting all physiology, but on zooplankton grazing as well.

A comparison of seasonal changes of phytoplankton pigment (this study) and mesozooplankton (Rudjakov et al. 1995) biomass values is given in Table 3, summarizing the approximate times of biomass maxima and minima. At higher latitudes the seasons of maximal and minimal phyto- and zooplankton abundances coincide approximately, with an accuracy of a month. If the data at hand allow more precise conclusions, in the Antarctic zone the phytoplankton maximum precedes the peak of mesozooplankton abundance, but, in

This article was presented by K. Banse, Seattle, Washington, USA contrast, follows it in the higher latitudes of the Northern Hemisphere: these patterns result in production types I and II identified by Heinrich (1993) for Antarctic and offshore regions of the boreal Pacific, respectively. At intermediate latitudes, the seasonal cycles of phyto-and zooplankton abundance differ greatly. The cause-and-effect relations between them cannot be traced intuitively. The processes of phyto- and zooplankton interactions are hardly understandable without the development of a mathematical model.

Acknowledgements. The work was supported by the Russian Foundation for Basic Research (95-04-12520). The statistical analysis of the data and the manuscript preparation were done at the Alfred Wegener Institute for Polar and Marine Research (AWI), Bremerhaven, Germany. I thank Prof. Victor Smetacek and Dr Ulrich Bathmann for the use of facilities at AWI Biology II laboratory and Prof. Karl Banse, University of Washington, for helpful advice, discussion and comments on the manuscript. This paper benefited from critical reading by Dr Richard Crawtord at AWI and 2 anonymous reviewers. Many thanks are due to Dr Athea Heinrich, P. P. Shirshov Institute of Oceanology, Moscow, Russia, for also reading the MS and discussing-even if not sharing-my ideas. This is AWI publication No. 1174

\section{LITERATURE CITED}

Banse K, English DC (1994) Seasonality of coastal zone color scanner phytoplankton pigment in the offshore oceans J Geophys Res 99:7323-7345

Bliss CI (1958) Periodic regression in biology and climatology Bull Conn Agr Exp Station, New Haven 615:1-55

Fukuchi M, Tanimura A, Ohtsuka H, Hoshiai T (1985) Marine biological data of Biomass Programme at Syowa Station in the 1982 winter (JARE-23). Part 1. Oceanographic data JARE Data Rept Nat Inst Polar Res, Tokyo 98:9-55

Heinrich AK (1993) Comparative ecology of the plankton oceanic communities. Nauka, Moscow (in Russian)

Holmes RW (1957) Solar radiation, submarine daylight, and photosynthesis. In: Hedgpeth JW (ed) Treatise on marine ecology and paleoecology 1. National Research Council, New York, p 109-128

Rudjakov JA, Tseitlin VB, Kitain VJ (1995) Seasonal variations of mesoplankton biomass in the upper layer of the Bering Sea; understanding biomass oscillations in the ocean. ICES J Mar Sci 52:747-753

Semina GI (1977) Phytoplankton. In: Vinogradov ME (ed) Oceanology - biology of the ocean. 1. Biological structure of the ocean. Nauka, Moscow, p 58-62 (in Russian)

Yoder JA, McClain CR, Feldman GF, Esaias WE (1993) Annual cycles of phytoplankton chlorophyll concentrations in the global ocean: a satellite view. Global Biogeochem Cycles 7:181-193

Manuscript first received: January 10, 1996

Revised version accepted: September 20, 1996 